Shock interactions with heavy gaseous elliptic cylinders: Two leeward-side shock competition modes and a heuristic model for interfacial circulation deposition at early times

Jaideep RayRavi SamtaneyNorman J. Zabusky

Citation: Physics of Fluids 12, 707 (2000); doi: 10.1063/1.870276

View online: http://dx.doi.org/10.1063/1.870276

View Table of Contents: http://aip.scitation.org/toc/phf/12/3

Published by the American Institute of Physics

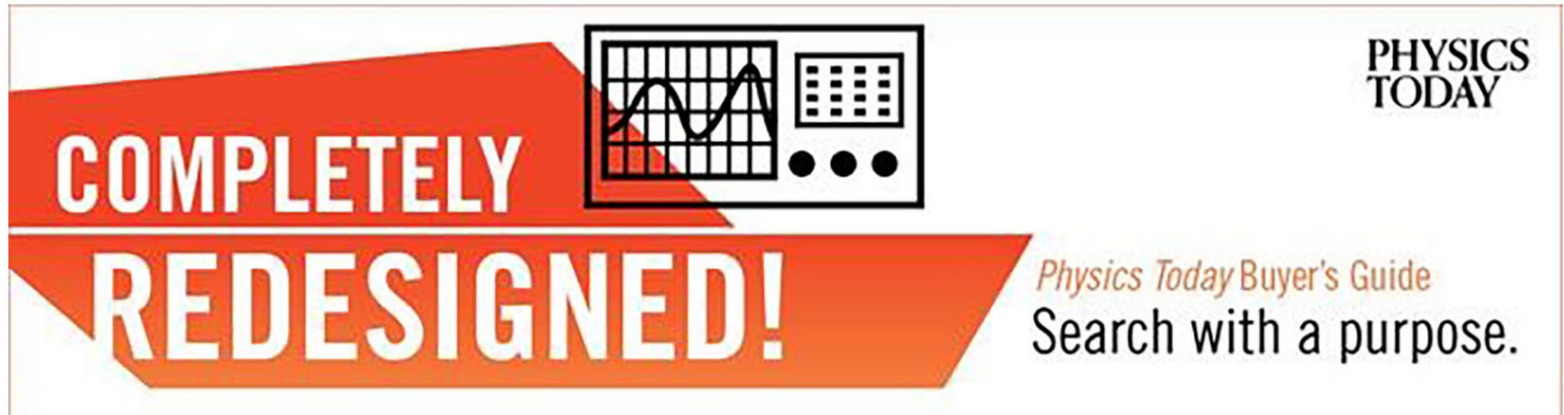




\title{
Shock interactions with heavy gaseous elliptic cylinders: Two leeward-side shock competition modes and a heuristic model for interfacial circulation deposition at early times
}

\author{
Jaideep Ray ${ }^{\mathrm{a}}$ \\ Department of Mechanical and Aerospace Engineering, 98 Brett Road, Rutgers University, Piscataway, \\ New Jersey 08854-8058 \\ Ravi Samtaney \\ Mail Stop 205-45, Graduate Aeronautical Laboratories, California Institute of Technology, Pasadena, \\ California 91125 \\ Norman J. Zabusky \\ Department of Mechanical and Aerospace Engineering, 98 Brett Road, Rutgers University, Piscataway, \\ New Jersey 08854-8058
}

(Received 17 August 1998; accepted 15 November 1999)

\begin{abstract}
We identify two different modes, types I and II, of the interaction for planar shocks accelerating heavy prolate gaseous ellipses. These modes arise from different interactions of the incident shock (IS) and transmitted shock (TS) on the leeward side of the ellipse. A time ratio $t_{T} /$ $t_{I}\left(M, \eta, \lambda, \gamma_{0}, \gamma_{b}\right)$, which characterizes the mode of interaction, is derived heuristically. Here, the principal parameters governing the interaction are the Mach number of the shock $(M)$, the ratio of the density of the ellipse to the ambient gas density, $(\eta>1), \gamma_{0}, \gamma_{b}$ (the ratios of specific heats of the two gases), $\lambda$ (the aspect ratio). Salient events in shock-ellipse interactions are identified and correlated with their signatures in circulation budgets and on-axis space-time pressure diagrams. The two modes yield different mechanisms of the baroclinic vorticity generation. We present a heuristic model for the net baroclinic circulation generated on the interface at the end of the early-time phase by both the IS and TS and validate the model via numerical simulations of the Euler equations. In the range $1.2 \leqslant M \leqslant 3.5,1.54 \leqslant \eta \leqslant 5.04$, and $\lambda=1.5$ and 3.0 , our model predicts the baroclinic circulation on the interface within a band of $\pm 10 \%$ in comparison to converged numerical simulations. (c) 2000 American Institute of Physics. [S1070-6631(00)00303-2]
\end{abstract}

\section{INTRODUCTION}

Accelerated inhomogeneous flows are everpresent. For example, the interaction of a shock with a density-stratified interface is a canonical problem in compressible hydrodynamics. ${ }^{1}$ Such studies ${ }^{2}$ are motivated by a desire to understand the baroclinic generation of vorticity and turbulent mixing in scramjets, inertial confinement fusion systems, and the astrophysical environments of planetary nebulas and supernovas.

In this paper, we focus on the interaction of a planar shock with a prolate, heavy (i.e., heavier than ambient) gas ellipse (elliptical cylinder), as shown in Fig. 1. Because of symmetry, we show only the top half. An incident shock (IS) of Mach number $M$, propagates from the left with a front perpendicular to the $x$ axis in an infinite gaseous medium of density $\rho_{0}$, pressure $p_{0}$, and ratio of specific heats $\gamma_{0}$, and strikes a prolate heavy gas ellipse of aspect ratio $\lambda$ and minor axis $2 b$, density $\rho_{b}$, pressure $p_{b}$, and ratio of specific heats $\gamma_{b}$. By prolate, we mean the minor axis of the ellipse is

\footnotetext{
${ }^{a}$ Corresponding author. Present address: Reacting Flow Research, Sandia National Laboratories, P.O. Box 969, MS 9056, Livermore, California 94551-0969. Phone: (925)-294-3638; fax: (925)-294-2595.
}

along the $x$ axis, the direction of propagation of the incident shock front. The parameters for the gases used in this investigation are shown in Table I.

When IS strikes the interface between the elliptical bubble and the ambient gas, it refracts into a transmitted shock (TS) and a reflected wave. Two generic classes of interactions exist: one where the IS moves faster than the TS (fast-slow or $\mathrm{f} / \mathrm{s}$ ), and vice versa (slow-fast or $\mathrm{s} / \mathrm{f}$ ). For the parameters considered in this paper, $\mathrm{f} / \mathrm{s}(\mathrm{s} / \mathrm{f})$ interactions are observed when $\eta=\rho_{b} / \rho_{0}>1(\eta<1)$. The reflected wave is usually a shock for a $\mathrm{f} / \mathrm{s}$ interaction and a rarefaction for s/f. If the IS, TS, and the reflected waves meet at a node on the interface, the refraction is called regular.

As the incident shock traverses the windward side of the elliptical interface, it generates a layer of vorticity baroclinically. However, on the leeward side, we identify new, more complex modes of circulation generation, associated with shock interactions, both on and off the interface. Essentially, the shock transmitted through the ellipse may reach and be transmitted through the leeward side before the IS completes its traversal of the leeward side. Our goal is to derive a heuristic model to quantify the baroclinic circulation deposited on the interface at the end of the early time phase, that is 


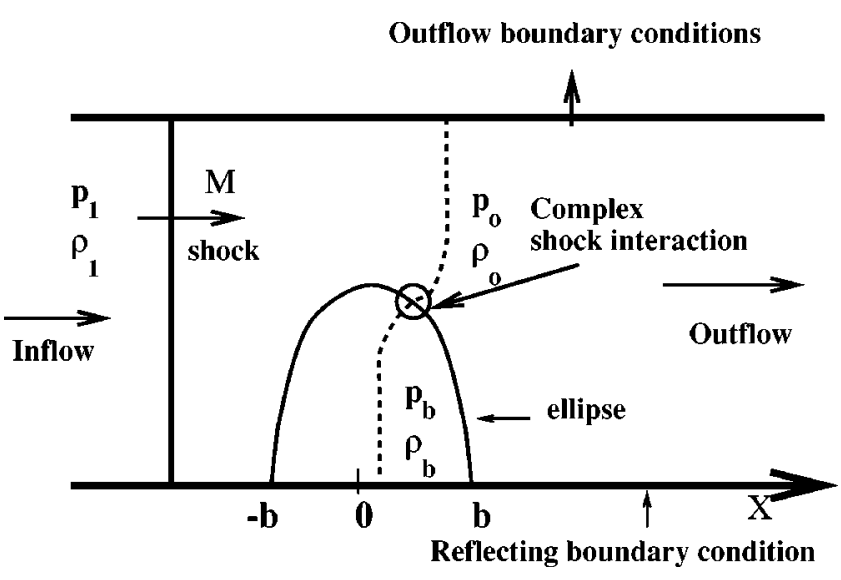

FIG. 1. Schematic of the physical setup. Due to symmetry, only the top half is shown. The solid vertical line shows the initial position of the shock. The dashed line shows the incident shock at a later time when it undergoes a complex shock refraction on the leeward side. This is indicated by the circle with a simple crossing of lines.

due to the traversal of the entire elliptical bubble by the incident and transmitted shocks.

Eventually, the dominant deposited circulation rolls up into two counter-rotating complex dipolar vortices that emerge from the interaction at late time, e.g., a time exceeding at least five ellipse passage times of the incident shock. These have been observed in simulations and experiments of low Mach number $M \leqslant 1.3$ shock interactions with circular cylinders, and their evolution may be explained in terms of incompressible stratified vortex dynamics.

Hawley and Zabusky ${ }^{3}$ and Yang et al. $^{4}$ were the first to emphasize and quantify vorticity in the evolution of shocked interfaces. Investigations of shock-accelerated circular cylinders were done experimentally by Haas and Sturtevant $(M$ $\leqslant 1.3)^{5}$ and Jacobs $(M \leqslant 1.15),{ }^{6}$ and numerically by Quirk and Karni $(M=1.22$, radius resolved by 450 grid cells $) .{ }^{7}$ For axisymmetric spheres, numerical simulations were done by McKee et al. $(M=10 \text {, radius resolved by } 240 \text { grid cells })^{8}$ and Zabusky and Zeng $(M=1.2,2.5$, and 5.0, radius resolved by 55 grid cells), ${ }^{9}$ who made numerous quantifications. Three-dimensional numerical simulations of shockellipsoid interactions were done by $\mathrm{Xu}$ and Stone $(M=10$, $\lambda=2$, major axis resolved by 128 grid cells). ${ }^{10}$ A starting point in the development of our model is the model for circulation deposition on heavy circular cylinders by Samtaney and Zabusky ${ }^{11}$ and baroclinic circulation quantifications for s/f interfaces by Samtaney et al. ${ }^{12}$

TABLE I. Gas pairs. Here $\eta$ is the preshocked density ratio, $\mathrm{At}=(\eta$ $-1) /(\eta+1)$ the preshock Atwood number, and $\gamma_{0}, \gamma_{b}$ are the specific heat ratios of the two gases.

\begin{tabular}{|c|c|c|c|}
\hline Parameters & air- $-\mathrm{CO}_{2}$ & $\begin{array}{l}\text { Gas pairs } \\
\text { air-R22 }\end{array}$ & air- $\mathrm{SF}_{6}$ \\
\hline$\eta(\mathrm{At})$ & $1.54(0.212)$ & $3.00(0.5)$ & $5.04(0.6689)$ \\
\hline$\gamma_{0}, \gamma_{b}$ & $1.4,1.297$ & $1.4,1.172$ & $1.4,1.0935$ \\
\hline
\end{tabular}

\section{NUMERICAL SIMULATIONS}

\section{A. Governing equations}

We first present results from numerical simulations to demonstrate the shock-competition mentioned above. Since viscous effects are negligible during the vorticity deposition phase of the shock-ellipse interaction, we adopt an inviscid model for simulation purposes. We make the following assumptions: the flow is inviscid, the gases are perfect, and there are no chemical reactions between the two gases, which are further assumed to be in thermal equilibrium. The governing equations (the compressible Euler equations) in conservative form are

$$
\mathbf{U}_{t}+\mathcal{F}(\mathbf{U})_{x}+\mathcal{G}(\mathbf{U})_{y}=0,
$$

where

$$
\begin{aligned}
& \mathbf{U}=\{\rho, \rho u, \rho \mathrm{v}, E, \rho \zeta\}^{T}, \\
& \mathcal{F}(\mathbf{U})=\left[\rho u, \rho u^{2}+p, \rho u \mathrm{v},(E+p) u, \rho \zeta u\right]^{T}, \\
& \mathcal{G}(\mathbf{U})=\left[\rho \mathrm{v}, \rho u \mathrm{v}, \rho \mathrm{v}^{2}+p,(E+p) \mathrm{v}, \rho \zeta \mathrm{v}\right]^{T},
\end{aligned}
$$

and $E$ is the total energy, related to the pressure $p$ by $p$ $=(\gamma-1)\left[E-\frac{1}{2} \rho\left(u^{2}+\mathrm{v}^{2}\right)\right]$.

In the above equations, the scalar quantity $\zeta(\mathbf{x}, t)$, defined as the volume fraction of the incident gas, is used to track the interface between the incident and transmitted gases. Here $\zeta(\mathbf{x}, t) \in[0,1]$ and the level set $\zeta(\mathbf{x}, t)=0.5$ is chosen to define the interface.

\section{B. Initial and boundary conditions}

The boundary conditions are post-incident shock values at the left boundary and quiescent flow $\left(p_{0}, \rho_{0}, \mathbf{u}=0\right)$ at the right boundary. Reflecting boundary conditions $(\mathbf{u} \cdot \mathbf{n}=0$, where $\mathbf{n}$ is the unit normal to the plane of the boundary) were enforced on the horizontal axis (axis of symmetry) and outflow boundary conditions were enforced on the top boundary. The ellipse is centered at the origin of the coordinate system, and because symmetry is assumed, only the top half of the ellipse is simulated. The initial condition for $\zeta$ is given by $\zeta(\mathbf{x}, 0)=1(0)$ in the incident (transmitted) gas. A shock moving in the positive $x$ direction is initialized a distance $X_{0}$ upstream of the ellipse using the Rankine-Hugoniot jump conditions.

\section{Numerical details}

Our numerical method is a second-order accurate Godunov scheme and includes interface tracking. A complete exposition of the numerical method can be found in our previous paper. ${ }^{12}$ The Godunov method gives rise to transverse oscillations behind the transmitted shock at high Mach numbers $(M>2.75)$ and high stratifications $(\eta>5)$, and, consequently, a second-order equilibrium flux method $(\mathrm{EFM})^{13}$ was used for them.

It should be noted that no explicit artificial viscosity was used in these numerical methods. However, these numerical methods do suffer from an implicit numerical viscosity that 
causes a local mixing of the incident and transmitted gases. The ratio of specific heats in a computational cell containing a mixture of the gases is calculated as

$$
\gamma=\frac{\gamma_{0} \zeta R_{0}+\gamma_{b}(1-\zeta) R_{b}}{\zeta R_{0}+(1-\zeta) R_{b}},
$$

where $R_{0}$ and $R_{b}$ are gas constants of the incident and transmitted gas, respectively. A uniform square $(\Delta x=\Delta y)$ mesh is used for all the simulations. Typically $X_{0}=10 \Delta x$. The interface is initially smeared over $2 \Delta x$ to $3 \Delta x$.

\section{Normalizations}

For simplicity, we assume $p_{b}=p_{0}=\rho_{0}=1$ and $\rho_{b}=\eta$. All length scales are normalized by $b$ (equivalent to specifying $b=1$ ), velocities by $c_{0}$, the speed of sound in the ambient medium (equivalent to specifying $c_{0}=1$ ), and time by $t^{*}=b / c_{0}$, the half-bubble traversal time by a linear acoustic wave.

\section{E. Validation}

Since we are modeling interfacial circulation, we validate the simulation codes as in our previous studies. ${ }^{12,14}$ The interfacial circulation $\Gamma_{\text {num }}$ is

$$
\Gamma_{\text {num }}=\sum_{D} \omega(i, j, t) \Delta x \Delta y,
$$

where $D=[(i, j, t) \forall \zeta(i, j, t): 0.001 \leqslant \zeta(\mathbf{x}, t) \leqslant 0.999]$ and the vorticity is

$$
\begin{aligned}
\omega(i, j, t)= & \frac{\mathrm{v}(i+1, j, t)-\mathrm{v}(i-1, j, t)}{2 \Delta x} \\
& -\frac{u(i, j+1, t)-u(i, j-1, t)}{2 \Delta y} .
\end{aligned}
$$

In Fig. 2 we establish convergence with respect to grid refinement for $\Gamma_{\text {num }}$ deposited on an ellipse. We plot the circulation for the parameter set $M=1.5, \eta=3.0, \lambda=1.5$, $\gamma_{0}=1.4, \gamma_{b}=1.172$, normalized by $M c_{0} b$, as a function of normalized time. For our early-time interval, we observe convergence for the interfacial circulation when the major axis of the ellipse was resolved by 180, 360, and 720 grid points. For the runs described in this paper, the major axis will be resolved by 360 grid cells.

\section{PHENOMENOLOGY FOR TWO MODES OF LEEWARD SIDE SHOCK-COMPETITION AND CIRCULATION DEPOSITION}

In this section, we distinguish between two modes (I and II) of shock-interface interaction that arise on the leeward side of a prolate ellipse. We introduce a shock-traversal time ratio that characterizes the appearance of the appropriate mode of the interaction.

\section{A. Classification}

\section{Type I}

Samtaney and Zabusky ${ }^{11}$ identified three phases in the interaction of shocks with circular cylinders. They are

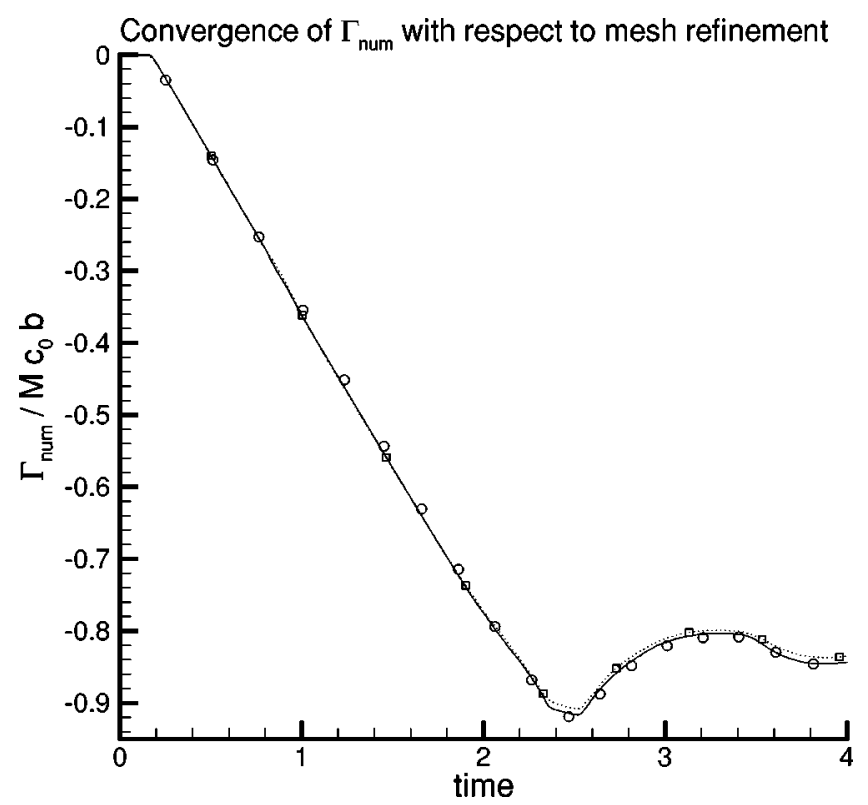

FIG. 2. Convergence study of baroclinic circulation deposited on the ellipse by the shock. The normalized circulation, $\left(\Gamma_{\text {num }} / M c_{0} b\right.$, is plotted as a function of normalized time. The parameters of the run are $M=1.5, \eta$ $=3.0, \lambda=1.5, \gamma_{0}=1.4, \gamma_{b}=1.172$. The major axis of the ellipse was resolved by 180 (dotted line with “ $\square$ ',), 360 (solid line), and 720 (" $\bigcirc$ ') grid points.

illustrated in Fig. 3, which shows five points on a prolate ellipse. Points A and D are the windward and leeward tips of the ellipse, respectively, while $\mathrm{B}$ is the crest. Point $\mathrm{C}$ $\left[\equiv\left(-x_{c}, y_{c}\right)\right]$ is the point where the shock refraction becomes irregular and $C^{\prime}$ is its mirror image on the leeward side. Both are essential to our heuristic model of early-time circulation deposition, as discussed below. By heuristic, we mean a phenomenological, nonasymptotic estimate of deposited circulation that is within a $\pm 10 \%$ band surrounding the numerically calculated value. The phases are the following.

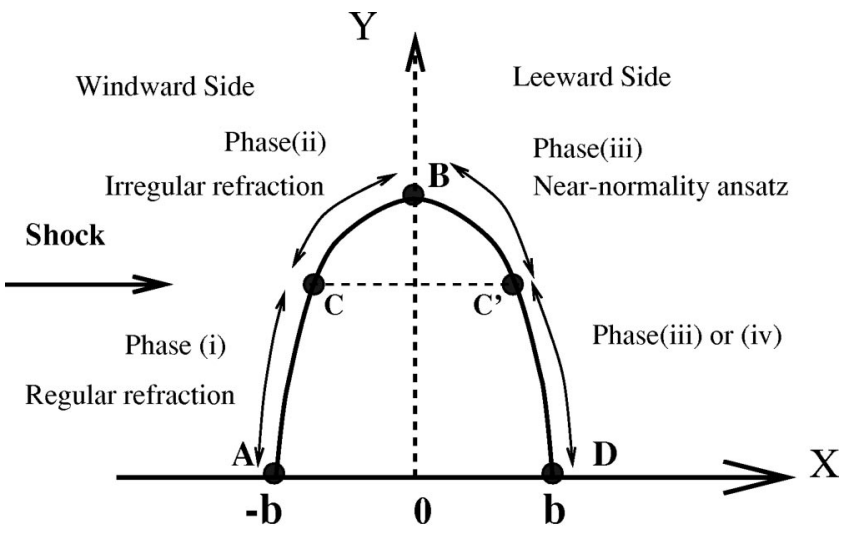

FIG. 3. The different phases in a shock-ellipse interaction. Here A and D are the windward and leeward tips of the ellipse, respectively, while $\mathrm{B}$ is the top. Point $\mathrm{C}\left[\equiv\left(-x_{c}, y_{c}\right)\right]$ is the point where the shock refraction becomes irregular. Here $\mathrm{C}^{\prime}$ is the mirror image on the leeward side. Phase (i) of the interaction occurs in $\mathrm{AC}$, phase (ii) in $\mathrm{CB}$, and phases (iii) and (iv) in $\mathrm{BC}^{\prime} \mathrm{D}$. Phase (i) is characterized by regular shock refraction and phase (ii) by irregular refraction. The near-normality ansatz is employed in phase (iii). Phase (iv) is observed only in type II interactions, and is characterized by shock competition and the TS interacting with the leeward interface $C^{\prime} D$. 


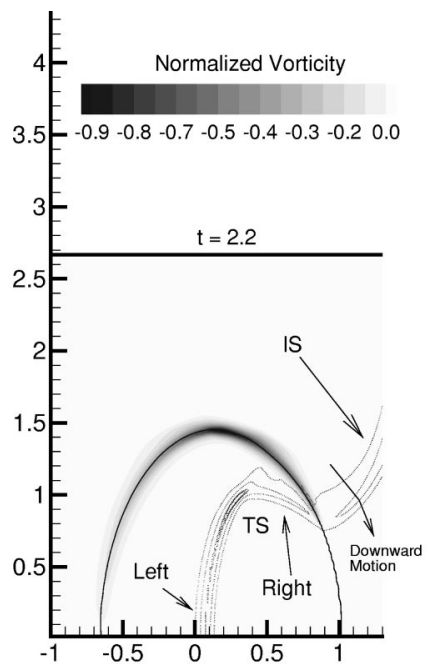

(a)

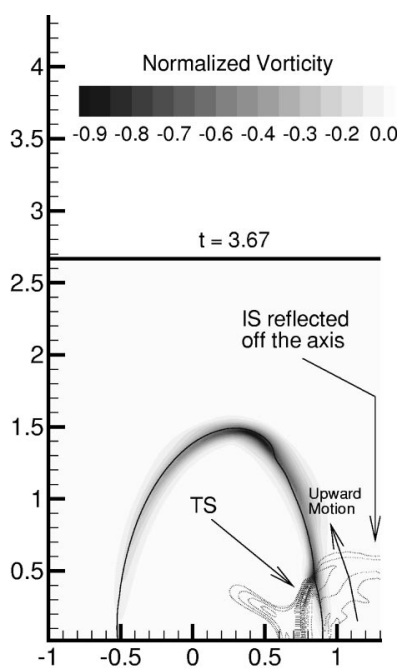

(b)
FIG. 4. A type I interaction at an air- $\mathrm{SF}_{6}$ (Table I) interface $(M=1.2, \lambda$ $=1.5)$. Divergence $(\nabla \cdot \mathbf{u})$ contours have been plotted by dotted lines on a normalized vorticity $\left(\omega / \omega_{\max }\right.$, where $\left.\omega_{\max }=\max (|\omega|)\right)$ field, which is given by the black-and-white color map. The interface $\zeta=0.5$ is also plotted using a solid line. In (a) we plot six $\nabla \cdot \mathbf{u}$ contours, equally spaced between -87 and +13 to elucidate the shock structures. We see the transmitted shock (TS) just before it undergoes a local s/f interaction with the leeward side of the bubble interface while IS traverses it. In (b) we plot 13 divergence contours, equally spaced between -130 and -3 . We see that the IS has reflected off the horizontal axis and is moving upward while TS undergoes a local s/f interaction with the leeward side of the ellipse. Other details can be found in Table II. Here $\omega_{\max } b / M c_{0}=8.62$, circulation deposition (numerical) at the end of the TS traversal: $\Gamma_{\text {num }} / M c_{0} b=0.863$. The simulation domain is $[-4.27: 4.27] \times[0: 2.667]$. The simulation was done using the Godunov scheme.

(i) Phase (i) : IS traverses AC on the windward side and undergoes regular refraction. This phase ends at $\mathrm{C}$ when $\alpha$, the local angle between the shock front and the ellipse, reaches the critical angle $\alpha_{\text {cr }}^{11}$ for regular refraction. The derivation for $\alpha_{\text {cr }}$ (as done in Ref. 11) is strictly applicable for planar oblique interfaces only and we employ it as an approximate measure of $\alpha_{\mathrm{cr}}$ for curved interfaces.

(ii) Phase (ii) : IS traverses CB and undergoes irregular refraction at the interface. This phase ends when IS reaches the crest point B, i.e., $\alpha=\pi / 2$.

(iii) Phase (iii) : IS traverses the leeward side of the ellipse (between B and D in Fig. 3). The incident shock expands around the top and bends back to meet the interface almost at $\alpha=\pi / 2 .{ }^{11}$ During this phase IS weakens, and if it is sufficiently weak, it transforms into a local region of compression waves near the interface. The effects of shock competition, as outlined below, are observed only in the BD section of the interface.

Figure 4 shows a Type $I$ interaction [in phase (iii)]. The results are from an $M=1.2, \eta=5.04, \lambda=1.5\left(\right.$ air- $\left.-\mathrm{SF}_{6}\right)$ simulation. The $\nabla \cdot \mathbf{u}$ (divergence) contours have been plotted over the normalized vorticity field $\left[\omega / \omega_{\max }\right.$, where $\left.\omega_{\max }=\max (|\omega|)\right]$ to juxtapose the shocks and the vorticity. The contour level $\zeta=0.5$ denotes the center of the interfacial layer and is seen as the dark line in Fig. 4. Figure 4(a) ( $t$ $=2.2$ ) shows the transmitted shock (TS) midway into the bubble. It has a nearly vertical left segment (approaching a

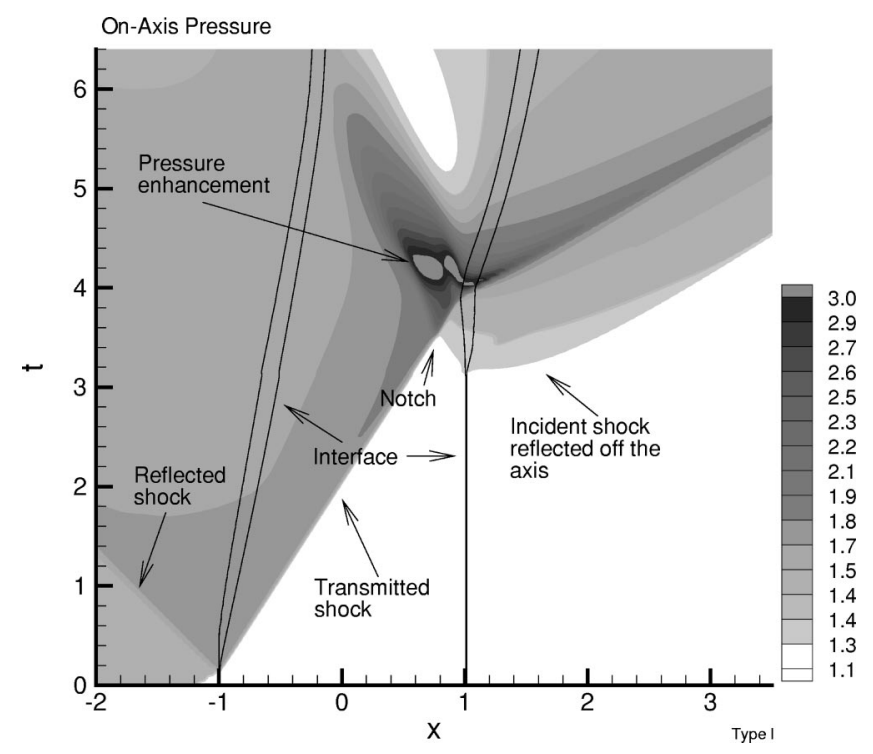

FIG. 5. The on-axis pressure plotted versus time for the simulation in Fig. 4. The locations of $\zeta=0.999$ and $\zeta=0.5$ limits on the $x$ axis have also been plotted. After the shock interaction on the windward side, there is a slow divergence between the $\zeta=0.999$ and $\zeta=0.5$ traces. This can also been seen on the leeward side at $t \approx 3.2$ when IS arrives at the $x$ axis. The notch at $t$ $\approx 3.55$ is formed as a result of the interaction of the TS and a wave sent upstream by the IS on reflecting off the axis. At $t \approx 4$ TS reaches the leeward side. We also see a pressure enhancement at $t \approx 4.2$.

local interaction with the leeward side) and a bent right segment that is connected to IS (which has nearly completed its traversal). The local interaction between the leeward side and TS can be $\mathrm{s} / \mathrm{f}$ or $\mathrm{f} / \mathrm{s}$. In this particular example it happens to be s/f. Figure 4(b) $(t=3.67)$ shows a s/f interaction between TS and the post-shocked ambient gas on the leeward side of the ellipse in progress while the IS reflects off the horizontal axis, depositing opposite-signed vorticity. Thus, TS completes its traversal of the ellipse after the IS. It is clear that there is circulation deposition first by the IS followed by circulation deposition by the TS. Note that IS compresses the ellipse, and therefore the length of the minor axis is smaller than 2. Furthermore, the shock imparts a mean velocity to the ellipse along the $x$ axis. Due to this the ellipse does not appear centered at the origin in Fig. 4.

Some of the interactions described above have their signatures in the plot of the on-axis pressure space-time plot (Fig. 5). The upstream and downstream limits of the interfacial region $(\zeta=0.999)$ and the nominal interface $(\zeta=0.5)$ on the $x$ axis have been plotted. After shock-interface interaction on the windward side, one sees a reflected and a transmitted shock, the latter approaching the leeward side of the ellipse. At the end of phase (iii), IS reflects off the $x$ axis (at $t=3.15$ ), sending a shock upstream into the bubble. This interacts with the left (nearly vertical) segment of TS (seen as a "notch" at $t=3.55$ and previously observed by Zabusky and Zeng" in axisymmetric spherical bubbles). Figure 4(b) shows the shock configurations a little later at $t=3.67$. Note that the right segment of TS has already undergone a s/f interaction on the leeward side and the left (nearly vertical segment) is in the process of doing so. Note, also, that Fig. 5 shows an enhancement of pressure at around $t=4.2$, similar 


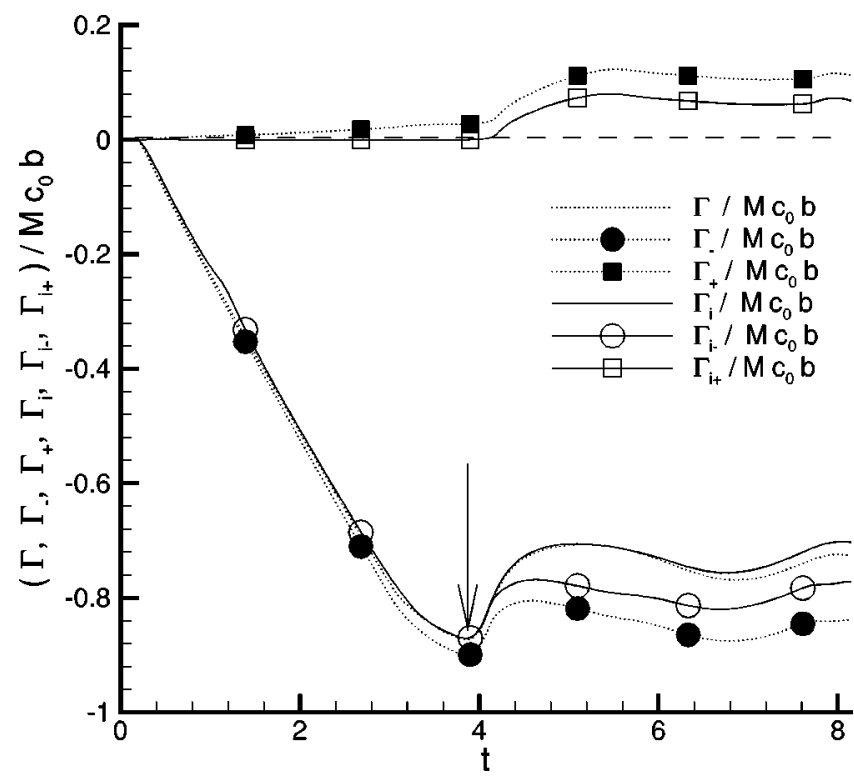

FIG. 6. Circulation budgets for the simulation shown in Fig. 4. Here $M$ $=1.2, \lambda=1.5$, and the gases are air and $\mathrm{SF}_{6}$. The dotted line shows the net global circulation, the dotted line with " " the negative circulation and the dotted line with " $\mathbf{\square}$ ' the positive circulation. The solid line shows the net interfacial circulation, the solid line with " $\bigcirc$ " the negative interfacial circulation, and the solid line with " $\square$ " the positive interfacial circulation. The arrow points to the value of circulation $\left(\Gamma_{i} / M c_{0} b\right)$ that is modeled.

to the cavity-collapse pressure enhancement seen by Zabusky and Zeng. ${ }^{9}$ A future study will elaborate this pressure enhancement.

Another view of these actions is shown in Fig. 6, showing the evolution of circulation. Here, we plot the net, positive, and negative budgets of the interfacial and global circulations. The important times are shown in Table II. $t_{\text {IS }}$, estimated from the simulation data, is the total elapsed time required by IS, from the start to reach the $x$ axis on the

TABLE II. Total elapsed times for salient events during shock traversal for type I and II interactions shown in Figs. 4-6 and Figs. 7-9, respectively. Times are: the following $t_{\text {contact }}$ is the time elapsed for IS before contact is made with the windward edge of the ellipse; $t_{\text {critical }}$ is the time elapsed for the IS to reach a point when the interaction becomes irregular; $t_{\text {crest }}$ is the time elapsed for the IS to reach the crest of the ellipse; $t_{\mathrm{IS}}$ the time elapsed for the IS to complete its traversal of the entire interface; and $t_{\mathrm{TS}}$ is the elapsed time for the TS to complete its traversal of the minor axis of the ellipse. Note that $t_{\mathrm{IS}}$ is estimated from the numerical data (Fig. 5) for type I interactions and analytically for type II. Here $t_{\mathrm{TS}}$ is estimated from numerical results (Figs. 5 and 8). All times have been normalized by $t^{*}=b / c_{0}$, the time needed by a linear acoustic wave to travel the semiminor axis of the ellipse. Also, $y_{c}$ is the height of the "stalk" of TS, estimated from the point where the interaction becomes irregular, i.e., $\alpha=\alpha_{\mathrm{cr}}$.

\begin{tabular}{lcc}
\hline \hline \multicolumn{1}{c}{ Figure No. } & 4 & 7 \\
\hline Parameters $(M, \lambda$, gas pair) & $1.2,1.5$, air-SF & $2.75,3.00$, air-R22 \\
Type of interaction & Type I & Type II \\
$t_{\text {contact }}$ & 0.18 & 0.18 \\
$t_{\text {crit }}$ & 0.75 & 0.46 \\
$t_{\text {crest }}$ & 1.00 & 0.54 \\
$t_{\text {IS }}$ (data,estimate) & 3.0 & 1.76 \\
$t_{\text {TS }}$ (data) & 3.95 & 1.20 \\
$y_{c}$ (estimate) & 1.4 & 2.92 \\
\hline \hline
\end{tabular}
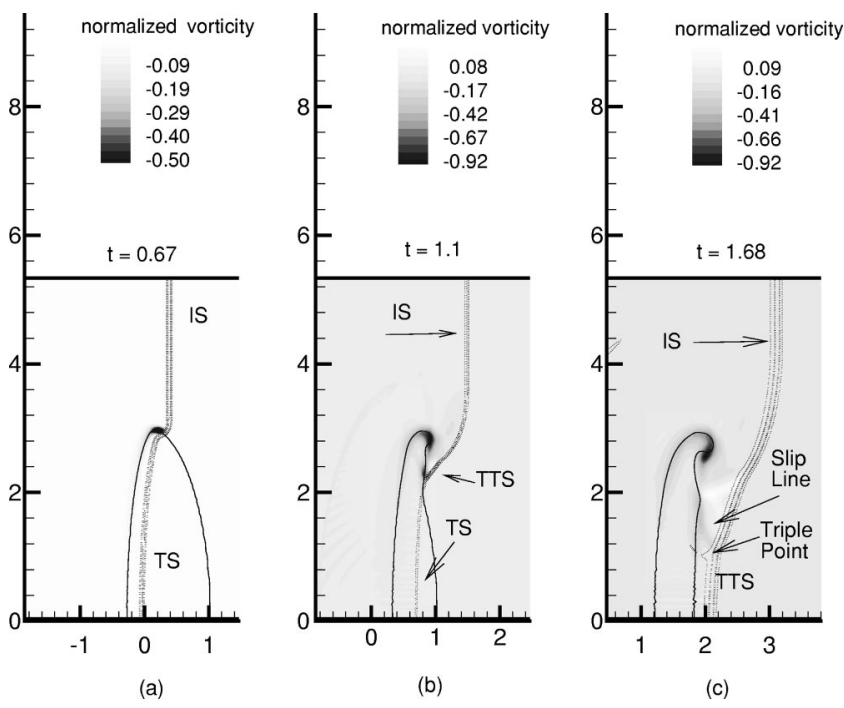

FIG. 7. A type II interaction at an air-R22 (Table I) interface ( $M$ $=2.75, \lambda=3.0)$. Selectively chosen divergence $(\nabla \cdot \mathbf{u})$ contours (dotted lines) have been overlaid on a normalized vorticity $\left(\omega / \omega_{\max }\right.$, where $\omega_{\max }$ $=\max (|\omega|))$ field to indicate the shocks. The interface $\zeta=0.5$ is plotted using a solid line. In (a) $(t=0.675)$ we see the transmitted shock (TS) just before it interacts with the incident shock (IS). In (b) $(t=1.1)$ the interaction is underway. In (c) $(t=1.68)$ we see that the transmitted shock has traversed through the ellipse before the incident shock. We also see a slip line emanating from a triple point on the TSS, formed as a result of the TTS-IS interaction on the leeward side. Here $\omega_{\max } b / M c_{0}=24.19$, circulation deposition (numerical) at the end of the TS traversal: $\Gamma_{\text {num }} / M c_{0} b=1.04$. For this simulation, the domain is $[-8.5: 8.5] \times[0: 5.333]$. The simulation was done using the Godunov scheme.

leeward side. Similarly, $t_{\mathrm{TS}}$ (also estimated from the numerical data) is the total elapsed time from start for the TS to cross the bubble interior and reach the leeward side. We see the expected linear growth and saturation of dominant negative circulation and the sudden growth of significant positive circulation $^{9}$ at $t \approx 4$ when the on-axis pressure enhancement reaches the leeward side of the ellipse. First we see a nearly linear rise in dominant negative circulation, with a slight rise in positive circulation after point $\mathrm{C}$ (Fig. 3), when the refraction becomes irregular. After $t=3.2$, the net negative circulation on the interface begins to saturate because of the upward-going IS and its magnitude suddenly declines at around $t=4.0$. We conjecture that this is due to the strong pressure wave following the collapse of the internal cavity (seen as the pressure enhancement in Fig. 5), as described in Ref. 9, depositing positive circulation on the leeward interface. The arrow at $t=4.0$ points to the net circulation used in comparing to the model. We note that at $t=8$ the positive and total interfacial circulation are around $8 \%$ and $81 \%$ of this value in magnitude.

\section{Type II}

Figure 7 shows $\nabla \cdot \mathbf{u}$ (divergence) contours, the mean interface location ( $\zeta=0.5$ contour), and the normalized vorticity field at three different times for a type II interaction with $M=2.75, \eta=3.0$, and $\lambda=3.0$ (air-R22). The TS traverses the ellipse before the IS and interacts with it on the leeward side. Thus the circulation deposited by IS is prema- 


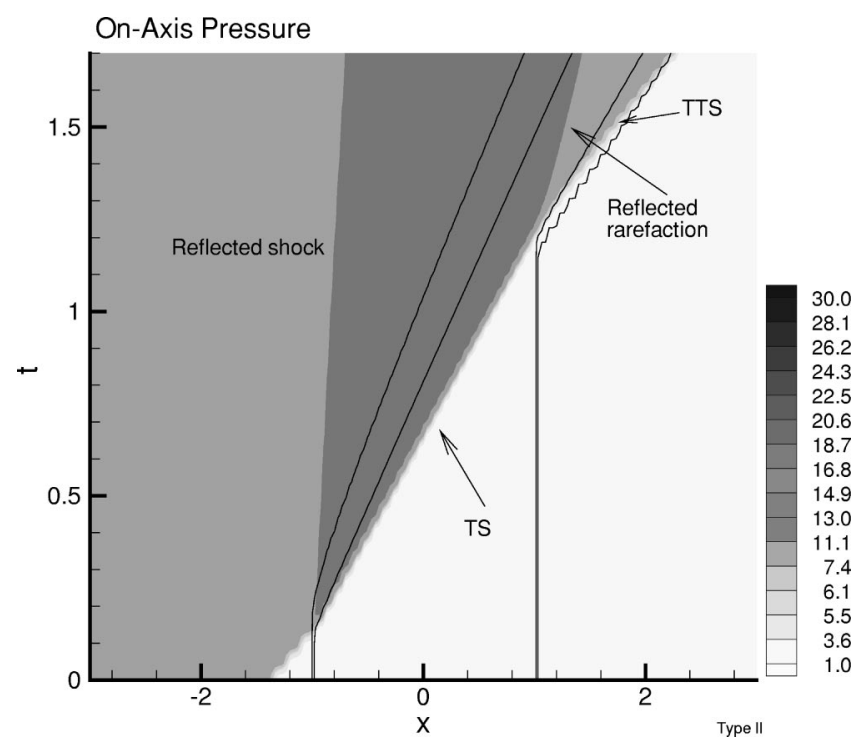

FIG. 8. The on-axis pressure plotted versus time for the simulation in Fig. 7. The locations of $\zeta=0.999$ and $\zeta=0.5$ limits on the $x$ axis have also been plotted. The two limits diverge, indicating the numerical diffusion of the initially sharp interface. We see the transmitted shock from the shock interaction on the windward side interacting with the leeward side at $t \approx 1.2$, giving rise to a reflected rarefaction. The on-axis complexities seen in Fig. 5 are absent here.

turely terminated and TS deposits circulation by a s/f interaction. In Fig 7(a) we see the incident shock (IS) traversing around the leeward side depositing negative vorticity, while the transmitted shock (TS) approaches the leeward interface. The nearly vertical left segment or "stalk" of the TS is about to undergo a local s/f interaction. In Fig. 7(b), we see TS undergoing a local s/f interaction with the unshocked ambient gas on the leeward side of the ellipse. It generates a transmitted shock (TTS, moving into the ambient) and a reflected rarefaction. We observe a complex shock system created by the TTS-IS interaction. Vorticity generation on the interface by the IS is terminated. In Fig. 7(c), we see that the TS has completed its traversal through the ellipse (and its interaction with the leeward side of the ellipse) and emerged from the bubble as TTS. Its interaction with the IS occurs off the interface. A slip line, formed as a result of the TTS-IS interaction, is seen emanating from a triple point on TS. We also see the incipient rolling up of the interface, as discussed in Ref. 15. Note, as in the previous interaction, the ellipse is compressed by the shock and translates along the $x$ axis.

Thus, type II can be said to have four phases, the first three being the same as in type I. Figure 7(a) shows phase (iii). The fourth phase commences when the "stalk" of the TS starts interacting with the leeward side at about $\mathrm{C}^{\prime}$ [Fig. 7(b)]. In Fig. 7(c), phase (iv) has ended, TTS has emerged from the bubble to interact with IS away from the interface. The existence of phase (iv) can be seen in the on-axis pressure diagram (Fig. 8), where neither the notch nor the pressure enhancement of type I are seen. Instead, one sees a s/f interaction of the TS at $t=1.2$, resulting in a reflected rarefaction and a TTS.

From the above discussion, in essence the IS and the TS compete on the leeward side of the ellipse. It is precisely this

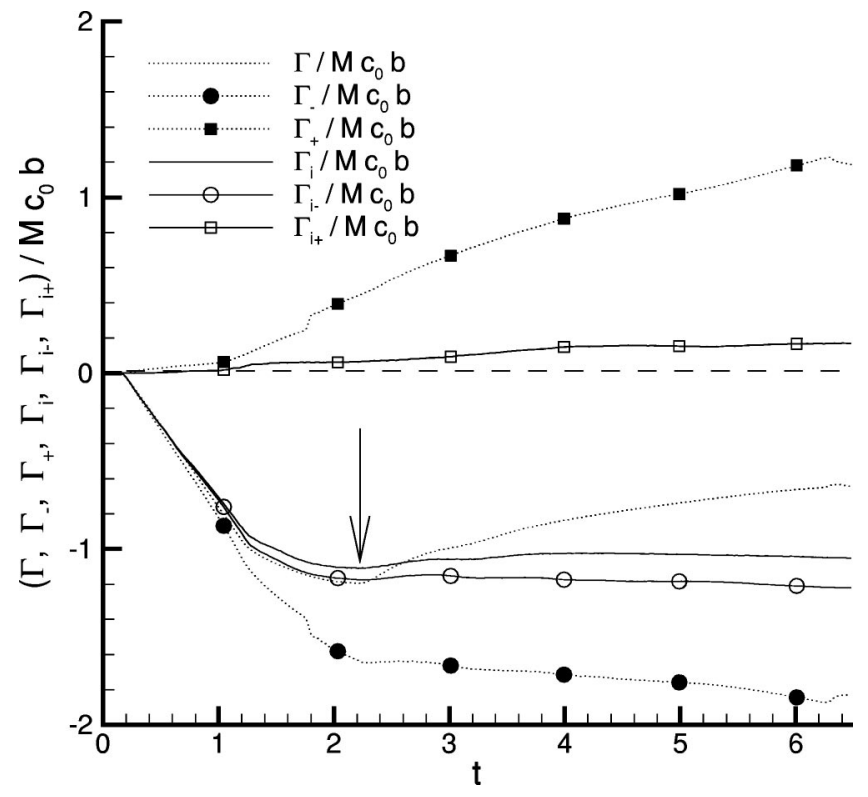

FIG. 9. Circulation budgets for the simulation shown in Fig. 7. Here $M$ $=2.75, \lambda=3.0$, and the gases are air and R22. The dotted line shows the net global circulation, the dotted line with the filled " $O$ ' the negative circulation, and the dotted line with the filled " $\square$ " the positive circulation. The solid line shows the net interfacial circulation, the solid line with " $\bigcirc$ ", the negative interfacial circulation, and the solid line with " $\square$ " the positive interfacial circulation. The arrow points to the value of circulation $\left(\Gamma_{i} / M c_{0} b\right)$ that is modeled.

shock-competition that determines the vorticity deposition mechanism.

We examine the vortex consequences of this type II behavior in Fig. 9. Again we show six circulations, positive and negative, and the net on and off the interface. We see the expected early-time linear growth, but also a strong positive component growing off the interface at around $t=1.3$. This is associated with the breakthrough at about $\mathrm{C}^{\prime}$. Furthermore, at this time the negative interfacial component begins to saturate for the same reason.

The arrow at $t=2.3$ points to the circulation used in comparing to the model. We note that at $t=6.0$ the positive and negative circulations off the interface are substantial, an effect to be quantified and modeled.

Note, in both these examples the interfacial domain expands rapidly after shock passage, and the expansion is larger for larger Mach numbers, a troublesome numerical artifact associated with low-order numerical schemes. ${ }^{16}$ In Fig. 8 , around $t \approx 1.7$, the interfacial domain area (or volume per unit span) is comparable to the interior bubble domain area. This diffusive effect must be understood and quantified before further late-time studies of instability and mixing can be believed and accurately modeled.

\section{B. Critical time and aspect ratios}

We now characterize the beginning of shockcompetition by means of the shock traversal time. To determine which type of interaction occurs on the leeward side, we characterize the movements of the IS around the leeward side and TS through the bubble. Conservatively, if the verti- 
cal stalk of the transmitted shock arrives at the rightmost leeward interface point before the undiminished-strength (assumed for simplicity) IS arrives at that point, we have a type II interaction. To model these interactions, we make a few simplifying approximations.

(i) On the leeward side of the ellipse, we adopt the nearnormality ansatz, ${ }^{11}$ i.e., the IS is locally perpendicular to the interface and moves with undiminished speed. This approximation is poor for weak shocks.

(ii) We approximate the "stalk" of the TS as a plane shock of height $y_{c}$, where $\left(-x_{c}, y_{c}\right)$ is the point on the interface where the incident shock refraction becomes irregular. In an irregular refraction at an elliptical interface, the TS system consists of a nearly vertical "stalk," topped by a triple point and a complex shock system. By approximating the TS as a plane shock of height $y_{c}$, we ignore the triple point and the complicated shock system associated with it, which is the right segment of the TS in its simplest form.

(iii) We assume that the height and strength of the TS remain unchanged as it propagates through the inside of the ellipse.

We estimate the time taken by the IS to traverse the prolate elliptical interface by

$$
t_{\mathrm{I}}=\frac{1}{M}\left(\int_{A}^{B} d x+\int_{B}^{D} d l\right)=M^{-1}\left[1+\lambda \mathcal{E}\left(\sqrt{1-\lambda^{-2}}\right)\right]
$$

and for an oblate elliptical interface by

$$
t_{\mathrm{I}}=M^{-1}\left[1+\mathcal{E}\left(\sqrt{1-\lambda^{2}}\right)\right],
$$

where $d l$ is the infinitesimal arclength along the interface, points A, B, and D have been defined in Fig. $3, \mathcal{E}(k)$ is a complete elliptic integral of the second kind and has the asymptotic limits :

$$
\mathcal{E}(k)= \begin{cases}\frac{\pi}{2}, & \text { for } k \rightarrow 0, \\ 1, & \text { for } k \rightarrow 1 .\end{cases}
$$

The time taken by the TS to traverse the interior of the ellipse is estimated as

$$
t_{T}=\frac{2}{M_{T} c_{b}},
$$

where $c_{b}$ is the speed of sound in the unshocked ellipse normalized by $c_{0}$ and $M_{T}$ is the Mach number of the transmitted shock. Note that $M_{T}$ is calculated from a onedimensional f/s shock interaction (consult Ref. 11 for details). For prolate ellipses, the ratio

$$
\frac{t_{T}}{t_{\mathrm{I}}}=\frac{M}{M_{T}} \sqrt{\frac{\gamma_{0}}{\gamma_{b}}} \frac{2 \sqrt{\eta}}{1+\lambda \mathcal{E}\left(\sqrt{1-\lambda^{-2}}\right)},
$$

determines the type of interaction:

$$
\frac{t_{T}}{t_{\mathrm{I}}} \begin{cases}>1, & \text { for type I and, } \\ <1, & \text { for type II. }\end{cases}
$$

The corresponding expression for oblate ellipses can be similarly derived.
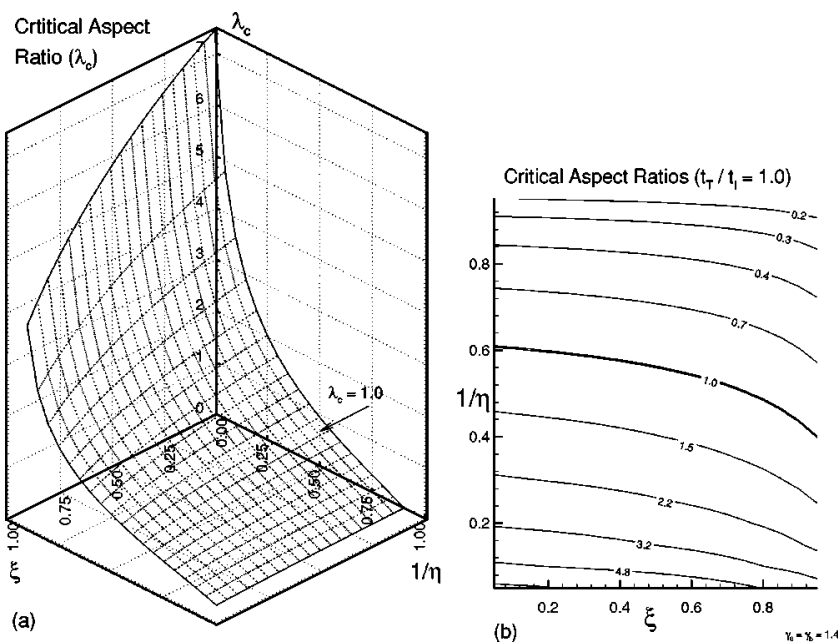

FIG. 10. Critical aspect ratio $\left[\lambda_{c}\right.$, Eq. (8) with $t_{T} / t_{\mathrm{I}}=1$ ) as a function of $1 / \eta$ and $\xi$, the normalized pressure gradient across the incident shock. $\gamma_{0}$ $=\gamma_{b}=1.4$. In (a) we plot the surface $\lambda=\lambda_{c}(\xi, \eta)$ to demarcate between type I and type II interaction spaces. For a type I interaction $\lambda<\lambda_{c}(\xi, \eta)$ (below the surface) and for a type II interaction $\lambda>\lambda_{c}(\xi, \eta)$ (above the surface). The $\lambda_{c}=1$ line has been darkened. In (b) we project the $\lambda_{c}$ surface to two dimensions. Ten exponentially spaced contours between 0.2 and 4.8 have been plotted. For a given $(\xi, \eta)$, if the $\lambda$ of the ellipse is lesser than $\lambda_{c}$, it undergoes a type I interaction.

We now derive an expression for a critical aspect ratio $\lambda_{c}$ such that type I or II processes prevail if $\lambda<\lambda_{c}$ or $\lambda$ $>\lambda_{c}$, respectively. If $\gamma_{0}=\gamma_{b}$ are fixed, then the 3-tuple $(\xi, \eta, \lambda)$ defines the parameter set for a given shock-ellipse interaction. Here, $\xi$ is the normalized pressure ratio across IS and is given by

$$
\xi(M)=\frac{[2 \gamma /(\gamma+1)]\left(M^{2}-1\right)}{1+[2 \gamma /(\gamma+1)]\left(M^{2}-1\right)},
$$

so that $\xi(M) \rightarrow 0$ (or 1) for weak (or strong) shocks, respectively. In Fig. 10(a), we plot the surface $\lambda=\lambda_{c}(\xi, \eta)$, the boundary between type I and II interactions, as a function of $\xi$ and $1 / \eta$ fixing the specific heat ratios $\left(\gamma_{0}=\gamma_{b}=1.4\right)$. An alternate presentation is given in Fig. 10(b), where lines of constant $\lambda_{c}$ are shown. Thus, for a given $\xi$ and $\eta$, if $\lambda$ $>\lambda_{c}$, then we have type II. This applies for oblate ellipses as well. We observe that in the $(\xi, \eta)$ space there is a region where $\lambda_{c}<1$. This implies that if only prolate ellipses ( $\lambda$ $>1$ ) are considered, then in this region one can only observe type II interactions.

For later use, we approximate the time $t_{c}$, for termination of primary circulation deposition by IS, as the time for TS to reach $\mathrm{C}^{\prime}$, or

$$
t_{c}=\frac{1+x_{c}}{M_{T} c_{b}} .
$$

\section{QUANTIFICATION AND MODELING OF INTERFACIAL BAROCLINIC CIRCULATION AT EARLY TIMES}

Samtaney et al. (Refs. 11 and 12) showed that the leading term in a series $[O(\sin \alpha)]$ for the normalized baroclinic circulation generated per unit length on a density-stratified 
interface is $\tilde{\sigma}_{1}=\sigma_{i}\left(M, \eta, \gamma_{0}, \gamma_{b}\right) \sin \alpha$, where $\alpha$ is the local angle between the shock front and the interface and subscript $i$ denotes either s/f or $\mathrm{f} / \mathrm{s}$. Equations for $\sigma_{i}\left(M, \eta, \gamma_{0}, \gamma_{b}\right)$, for both s/f and f/s interactions, are given in the Appendix for completeness. To obtain the circulation on the interface, we assume the IS strength $M$ remains constant and the tangent to the IS front is locally perpendicular to the interface as it diffracts around the leeward side of the bubble. Thus, in the absence of shock competition, the circulation deposited by IS on a heavy ellipse at the end of phase (iii) is modeled by

$$
\Gamma_{\mathrm{f} / \mathrm{s}}=\sigma_{\mathrm{f} / \mathrm{s}}\left(M, \eta, \gamma_{0}, \gamma_{b}\right)\left[1+\lambda \mathcal{E}\left(\sqrt{1-\lambda^{-2}}\right)\right] .
$$

Type I interactions (characterized by $t_{T} / t_{\mathrm{I}}>1$ ) contain a primary $\mathrm{f} / \mathrm{s}$ deposition by the IS and a secondary deposition by the TS. Thus

$$
\Gamma_{\mathrm{I}}=\Gamma_{\mathrm{f} / \mathrm{s}}+\sigma_{i}\left(M_{T}, 1 / \eta^{\prime}, \gamma_{b}, \gamma_{0}\right)\left(1-x_{c}\right),
$$

where $\eta^{\prime}=\rho_{b} / \rho_{0}^{\prime}, \rho_{0}^{\prime}$ is the post-shocked density of the ambient gas, approximated from a one-dimensional (1D) shock-interface interaction. Subscript " $i$ "' in Eq. (12) is either f/s or s/f, depending upon whether $\eta^{\prime}>1$ or $\eta^{\prime}<1$, respectively. Note, prior to shock-competition (i.e., during the primary deposition) it is observed in numerical simulations (and is a property of our first-order deposition model) that the time rate of baroclinic circulation deposition is a constant.

Type II interactions should also contain two circulation deposition terms. The first term is due to the (prematurely terminated) deposition by the IS (approximated by $\Gamma_{\mathrm{f} / \mathrm{s}} t_{c} / t_{\mathrm{I}}$ ), and the second term is the due to the s/f interaction of the TS at an interface of density ratio $1 / \eta$. Therefore, the total circulation deposited in a type II interaction is

$$
\Gamma_{\mathrm{II}}=\Gamma_{\mathrm{f} / \mathrm{s}} \frac{t_{c}}{t_{\mathrm{I}}}+\sigma_{\mathrm{s} / \mathrm{f}}\left(M_{T}, 1 / \eta, \gamma_{b}, \gamma_{0}\right)\left(1-x_{c}\right) .
$$

We quantify the interfacial circulation from the numerical simulations $\left(\Gamma_{\text {num }}\right)$ at the end of the TS traversal of the interior of the ellipse and plot $\Gamma_{\text {num }} / \Gamma_{\mathrm{I}}$ for $\eta=3.0, \lambda=1.5$ (air-R22) and $\eta=5.04, \lambda=1.5\left(\right.$ air- $\left.\mathrm{SF}_{6}\right)$ simulations in Fig. 11. In Fig. 12 we plot $\Gamma_{\text {num }} / \Gamma_{\text {I }}$ for $1.2 \leqslant M \leqslant 3.5$ for $\eta$ $=1.54, \lambda=1.5\left(\right.$ air $\left.-\mathrm{CO}_{2}\right)$ and $\eta=3.0, \lambda=3.0$ (air-R22). The difference in circulation deposition in numerical simulations and the model is less than $10 \%$ for both types of interactions. In Fig. 13 we plot $\Gamma_{\text {num }} / \Gamma_{\text {model }}$ for $M=1.5, \eta=3.0$ (air-R22) and $M=1.5, \eta=5.04\left(\right.$ air- $\left.\mathrm{SF}_{6}\right)$ interactions for different values of the aspect ratio, $\lambda \in[1,3]$. Here $\Gamma_{\text {model }}$ is calculated using Eq. (13) [Eq. (12)] for $t_{T} / t_{\mathrm{I}}<1 \quad\left(t_{T} / t_{\mathrm{I}}\right.$ $>1$ ), respectively. No clear trend is observed for the departure of the model from the numerical simulations. However, these departures are small (typically less than $\pm 5 \%$ ) and could be due to secondary phenomena, which our first-order model fails to capture in a region of complex flow physics.

\section{CONCLUSION}

The interaction of a shock with a heavy prolate ellipse is characterized by leeward-side complex shock interactions. These phenomena lead to two types of shock-ellipse interactions, referred to as type I and II. We use physical space

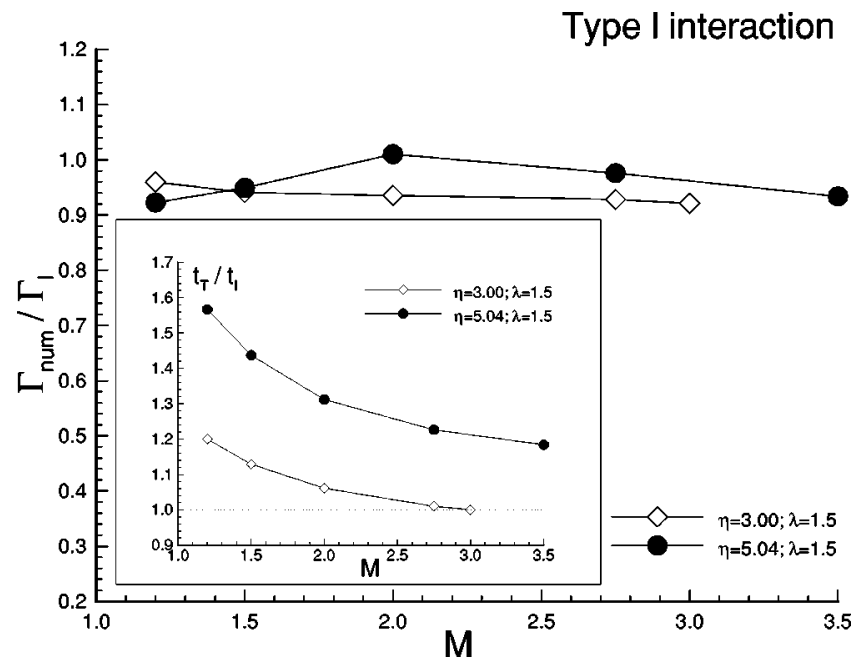

FIG. 11. Here $\Gamma_{\text {num }} / \Gamma_{\text {I }}$ [Eq. (12)] for $1.2 \leqslant M \leqslant 3.5$ for type I interactions. Results have been plotted for $\eta=3.0, \lambda=1.5$ (air-R22, " $\diamond "$ ) and $\eta$ $=5.04, \lambda=1.5$ (air-SF 6 , “”). Here $M$ is limited to 3.0 in the air-R22 case, since for $M>3.0$, for a $\lambda=1.5$ ellipse, the interaction becomes type II. Inset: $t_{T} / t_{\mathrm{I}}$ has been plotted for all the cases to show the type of interaction.

"snapshots" of velocity-divergence and vorticity, on-axis pressure space-time $(\mathrm{S} / \mathrm{T})$ diagrams and evolving circulation budgets to visualize shock-competition and circulation deposition.

In a type I interaction, the incident shock (IS) completes its traversal of the elliptical bubble before the transmitted shock (TS) does. IS reflects off the symmetry axis and sends an upstream shock through the ellipse, which, in turn, interacts with TS. This is seen as a prominent notch in the pressure S/T diagram (Fig. 5). In addition, the axis-reflected IS contributes to the circulation deposition on the interface.

In a type II interaction, the transmitted shock traverses through the ellipse and interacts with the leeward side before the incident shock does - seen clearly again in the on-axis pressure $\mathrm{S} / \mathrm{T}$ diagram as the TS interacts with the undis-

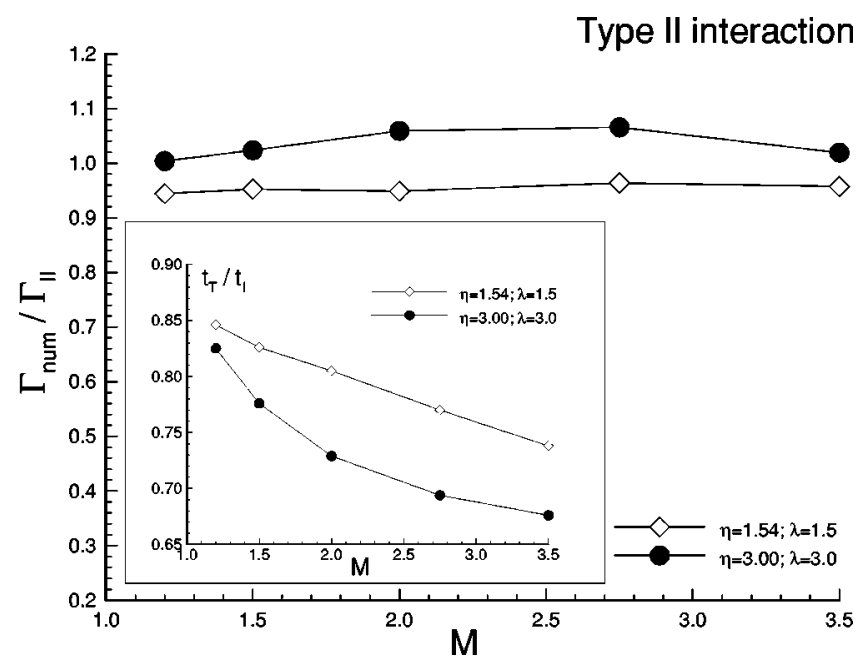

FIG. 12. Here $\Gamma_{\text {num }} / \Gamma_{\text {II }}$ [Eq. (13)] for $1.2 \leqslant M \leqslant 3.5$ for type II interactions. Results have been plotted for $\eta=1.54, \lambda=1.5$ (air- $\mathrm{CO}_{2}$, " $\bullet$ ") and $\eta$ $=3.0, \lambda=3.0$ (air-R22, " $\diamond ")$. Inset: $t_{T} / t_{\mathrm{I}}$ has been plotted for all the cases to show the type of interaction. 


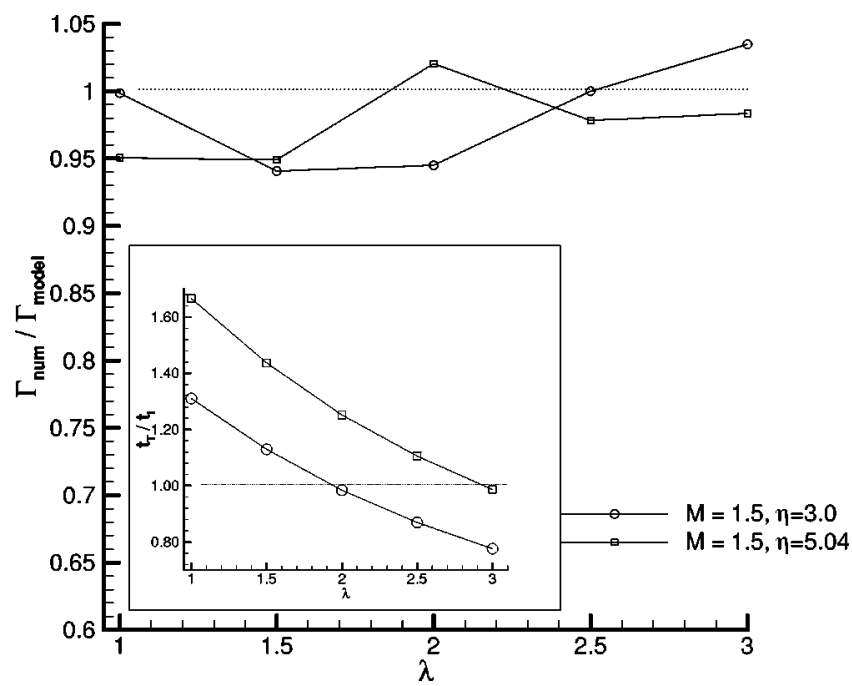

FIG. 13. Variation of $\Gamma_{\text {num }} / \Gamma_{\text {model }}$ vs $\lambda(1 \leqslant \lambda \leqslant 3)$ for $M=1.5, \eta=3.0$ (air-R22, " $\bigcirc$ ") and $M=1.5, \eta=5.04$ (air- $\mathrm{SF}_{6}$, " $\square$ ") interactions. Inset: $t_{T} / t_{\mathrm{I}}$ has been plotted for the two sets of data. Here $\Gamma_{\text {model }}$ has been calculated using Eq. (13) [Eq. (12)] for $t_{T} / t_{\mathrm{I}}<1\left(t_{T} / t_{\mathrm{I}}>1\right)$, respectively.

turbed leeward extremity of the bubble (Fig. 8). An approximate expression is given to represent the traversal time ratio to demarcate the two types of interactions. It is the ratio of the time taken for the IS to cover the entire interfacial circumference to the time taken for a planar TS to move across the elliptic bubbles interior. We also correlate certain salient events (e.g., the transition from regular to irregular shock refraction in the shock-ellipse interaction on the windward side), with their signatures in the positive, negative, and net circulation budgets. In addition to the details of shockellipse interactions, we find that, for a given shock strength and gas pair, there exists a critical aspect ratio of the elliptical cylinder for which the time ratio is unity. We show that for a certain region of the parameter space, prolate ellipses can experience only one type of interaction.

A heuristic model for the baroclinic circulation deposited on the interface at the end of early time (when both TS and IS have departed the leeward interface) is proposed and validated against converged numerical simulations. The model incorporates the effect of shock competition and its results fall within a $\pm 10 \%$ band about the numerical solution. For somewhat later times, the total positive and negative circulations, resulting in part from off-interface shock processes, may differ substantially from the results of the interfacial model.

\section{ACKNOWLEDGMENTS}

The computations were done on the T3E at the Pittsburgh Supercomputing Center and the Origin2000 at NCSA, University of Illinois, Urbana-Champaign. A portion of this work was done in Vizlab, part of which is supported by CAIP, Rutgers University. Ravi Samtaney gratefully acknowledges the support of the Data Analysis Group, NAS Division at the NASA Ames Research Center.

The work was supported by DOE under grants from Dr. D. Hitchcock and Dr. F. Howes.

\section{APPENDIX: CALCULATION OF THE CIRCULATION MODEL TERMS}

In this appendix, we provide, without details, sufficient information to calculate the terms $\left(\sigma_{\mathrm{s} / \mathrm{f}}\right.$ and $\left.\sigma_{\mathrm{f} / \mathrm{s}}\right)$ in the model derived in Sec. IV.

The baroclinic circulation generation per unit length of a fast-slow interface can be expressed as a series in $\sin \alpha$, where $\alpha$ is the local angle between the shock front and the interface. The coefficient of the first-order term is $\sigma_{\mathrm{f} / \mathrm{s}}\left(M, \eta, \gamma_{0}, \gamma_{b}\right)$ (derived in Ref. 11) is

$$
\begin{aligned}
\sigma_{\mathrm{f} / \mathrm{s}}= & \frac{1}{\gamma_{0}^{1 / 2} M}\left(\frac{\gamma_{b}}{\gamma_{b}-1} \frac{1-\psi\left(p_{20}, \mu_{b}\right)}{\eta}\right. \\
& \left.-\frac{\gamma_{0}}{\gamma_{0}-1}\left[1-\psi\left(p_{20} / p_{1}, \mu_{0}\right) \psi\left(p_{1}, \mu_{0}\right)\right]\right) .
\end{aligned}
$$

In the above equation, $p_{1}$ is the pressure behind the incident shock (of Mach number $M$ ) and is given by

$$
p_{1}=1+\frac{2 \gamma_{0}}{\gamma_{0}+1}\left(M^{2}-1\right) \text {. }
$$

Furthermore, $p_{20}$ is the pressure behind the reflected shock for $\alpha \rightarrow 0$ and may be calculated by solving the following nonlinear algebraic equation:

$$
\begin{gathered}
\frac{p_{1}-1}{\left(\mu_{0}^{2}+p_{1}\right)^{1 / 2}}-\frac{\left(p_{20} / p_{1}\right)-1}{\left(\mu_{0}^{2}+\left(p_{20} / p_{1}\right)\right)^{1 / 2}} \psi^{1 / 2}\left(p_{1}, \mu_{0}\right) \\
-\eta^{-1 / 2} \frac{p_{20}-1}{\left(\mu_{b}^{2}+p_{20}\right)^{1 / 2}}\left(\frac{\gamma_{0}+1}{\gamma_{b}+1}\right)^{1 / 2}=0 .
\end{gathered}
$$

The function $\psi(\xi, \mu) \equiv \xi\left[\left(1+\mu^{2} \xi\right) /\left(\mu^{2}+\xi\right)\right]$, and $\mu_{i}^{2}$ $=\left(\gamma_{i}-1\right) /\left(\gamma_{i}+1\right)$.

Likewise, for a slow-fast interface, one may express the baroclinic circulation generation as a series in $\sin \alpha$. The coefficient of the first term in the series, $\sigma_{\mathrm{s} / \mathrm{f}}\left(M, \eta, \gamma_{0}, \gamma_{b}\right)$ (derived in Ref. 12) is

$$
\begin{aligned}
\sigma_{\mathrm{s} / \mathrm{f}}= & \frac{1}{\gamma_{0}^{1 / 2} M}\left\{\frac{\gamma_{b}}{\gamma_{b}-1} \frac{1-\psi\left(p_{20}, \mu_{b}\right)}{\eta}\right. \\
& \left.-\frac{\gamma_{0}}{\gamma_{0}-1}\left[1-\left(\frac{p_{20}}{p_{1}}\right)^{\left(\gamma_{0}-1\right) / \gamma_{0}} \psi\left(p_{1}, \mu_{0}\right)\right]\right\} .
\end{aligned}
$$

In the above equation, $p_{20}$ is the pressure behind the transmitted shock for $\alpha \rightarrow 0$ and is calculated by solving the following nonlinear algebraic equation:

$$
\begin{gathered}
\frac{\left(1+\mu_{0}^{2}\right)^{1 / 2}}{\gamma_{0}} \frac{p_{1}-1}{\left(\mu_{0}^{2}+p_{1}\right)^{1 / 2}}+\frac{2}{\gamma_{0}-1} \psi^{1 / 2}\left(p_{1}, \mu_{0}\right) \\
\quad \times\left[1-\left(\frac{p_{20}}{p_{1}}\right)^{\left(\gamma_{0}-1\right) / 2 \gamma_{0}}\right] \\
=\frac{\left(1+\mu_{b}^{2}\right)^{1 / 2}}{\gamma_{b}\left(\eta \gamma_{0} / \gamma_{b}\right)^{1 / 2}} \frac{p_{20}-1}{\left(\mu_{b}^{2}+p_{20}\right)^{1 / 2}} .
\end{gathered}
$$


${ }^{1}$ N. J. Zabusky, "Vortex paradigm for accelerated inhomogeneous flows: Visiometrics for the Rayleigh-Taylor and Richtmyer-Meshkov environments," Annu. Rev. Fluid Mech. 31, 495 (1999).

${ }^{2}$ Proceedings of the 6th International Workshop on the Physics of Compressible Turbulent Mixing, 1998, edited by G. Jourdan and L. Houas, held at Marseilles, France, 18-21 June, 1997, Imprimerie Caractere, 26, Rue Saint Bruno, 13004 Marseilles, France.

${ }^{3}$ J. F. Hawley and N. J. Zabusky, "Vortex paradigm for shock-accelerated density-stratified interfaces," Phys. Rev. Lett. 63, 1241 (1989).

${ }^{4}$ X. Yang, I-L. Chern, N. J. Zabusky, R. Samtaney, and J. F. Hawley, "Vorticity generation and evolution in shock-accelerated density-stratified interfaces," Phys. Fluids A 4, 1531 (1992).

${ }^{5}$ J. F. Haas and B. Sturtevant, "Interaction of weak shock waves with cylindrical and spherical gas inhomogeneities," J. Fluid Mech. 181, 41 (1987).

${ }^{6} \mathrm{~J}$. W. Jacobs, "Shock induced mixing of a light-gas cylinder," J. Fluid Mech. 234, 629 (1992).

${ }^{7}$ J. J. Quirk and S. Karni, "On the dynamics of a shock-bubble interaction,'” J. Fluid Mech. 318, 129 (1996).

${ }^{8}$ R. Klein, C. F. McKee, and P. Colella, "'On the hydrodynamic interaction of shock waves with interstellar clouds I. Nonradiative shocks in small clouds," Astrophys. J. 420, 213 (1994).
${ }^{9}$ N. J. Zabusky and S. M. Zeng, "'Shock cavity implosion morphologies and vortical projectile generation in axisymmetric shock-spherical fast/slow bubble interactions," J. Fluid Mech. 362, 327 (1998).

${ }^{10}$ J. Xu and J. M. Stone, "The hydrodynamics of shock-cloud interactions in three dimensions," Astrophys. J. 454, 172 (1995).

${ }^{11}$ R. Samtaney and N. J. Zabusky, "Circulation deposition on shockaccelerated planar and curved density stratified interfaces: Models and scaling laws," J. Fluid Mech. 269, 45 (1994).

${ }^{12}$ R. Samtaney, J. Ray, and N. J. Zabusky, "Baroclinic circulation generation on shock accelerated slow/fast gas interfaces," Phys. Fluids 10, 1217 (1998).

${ }^{13}$ D. I. Pullin, "Direct simulation methods for compressible ideal gas flow," J. Comput. Phys. 34, 231 (1980).

${ }^{14}$ R. Samtaney and D. I. Meiron, "Hypervelocity Richtmyer-Meshkov instability," Phys. Fluids 9, 1783 (1997).

${ }^{15}$ R. Samtaney and D. I. Pullin, “'On initial-value and self-similar solutions of the compressible Euler equations," Phys. Fluids 8, 2650 (1996).

${ }^{16} \mathrm{~K}$. Xu, "Gas-kinetic schemes for unsteady compressible flows," von Karman Institute for Fluid Dynamics, Lecture Series 1998, 23-27 February, 1998. 